\title{
ALYREF Drives Cancer Cell Proliferation Through an ALYREF-MYC Positive Feedback Loop in Glioblastoma
}

This article was published in the following Dove Press journal: OncoTargets and Therapy

\author{
Jianjun Wang $\mathbb{1 D}^{1, *}$ \\ Yuchen $\mathrm{Li}^{2, *}$ \\ Binbin $\mathrm{Xu}^{3, *}$ \\ Jiao Dong' \\ Haiyan Zhao' \\ Dongxia Zhao' \\ Yong $\mathrm{Wu} \mathbb{D}^{4}$
}

'Department of Pediatric Medicine, Gansu Provincial People's Hospital, Lanzhou City 730000, Gansu Province, People's Republic of China; ${ }^{2}$ Fudan University Shanghai Cancer Center, Institute of Biomedical Sciences, Shanghai Medical College, Fudan University, Shanghai 200032, People's Republic of China; ${ }^{3}$ Department of Ophthalmology, Eye, Ear, Nose, and Throat Hospital, Fudan University, Shanghai, People's Republic of China; ${ }^{4}$ Department of Gynecologic Oncology, Fudan University Shanghai Cancer Center, Shanghai, People's Republic of China

*These authors contributed equally to this work
Background: While RNA-binding proteins (RBPs) are known to affect RNA homeostasis during cancer cell initiation and development, their characteristics and biological function in glioblastoma (GBM) remain unclear.

Methods: Differences in RBP expression were explored by differential analysis of The Cancer Genome Atlas-GBM and Genotype-Tissue Expression (GTEx) datasets. Real-time PCR was conducted to verify the expressional levels of Aly/REF export factor $(A L Y R E F)$ in normal brain and GBM tissues. Proliferative assays were performed to investigate molecular functions of ALYREF in GBM cells in vitro and in vivo. Real-time PCR and Kyoto Encyclopedia of Genes and Genomes (KEGG) were performed to analyze the ALYREF downstream signaling pathways. A chromatin immunoprecipitation (ChIP) assay was performed to identify key transcriptional factors that regulate $A L Y R E F$ expression at RNA level. UV crosslinking, immunoprecipitation (CLIP) and RNA stability assays were conducted to reveal the bound RNAs and their stability regulated by $A L Y R E F$.

Results: The results showed that $A L Y R E F$ is frequently increased in GBM tissues, and its mRNA expression is regulated by the MYC proto-oncogene, bHLH transcription factor (MYC). Inhibition of $A L Y R E F$ expression decreased GBM cell proliferative ability in vitro and tumor formation in vivo. KEGG analysis revealed that high $A L Y R E F$ expression in GBM tissues was enriched in the upregulation of oncogenic pathways such as the Wnt $/ \beta$-catenin signaling pathway. The CLIP assay showed that ALYREF drives GBM carcinogenesis by binding to and stabilizing $M Y C$ mRNAs. Overexpression of $M Y C$ restored the oncogenic property of $A L Y R E F$-deficient GBM cells.

Conclusion: Our data showed that $A L Y R E F$ is regulated by $M Y C$ at the transcriptional level. $A L Y R E F$ drives GBM cell proliferation by activating the $\mathrm{Wnt} / \beta$-catenin signaling pathway and stabilizing $M Y C$ mRNA, suggesting that an ALYREF-MYC positive feedback loop might be a potential therapeutic target for treating GBM patients.

Keywords: RNA-binding protein, MYC, cell proliferation, GBM

\section{Introduction}

Glioblastoma (GBM) is an aggressive tumor affecting the brain or spine. The median survival of GBM patients is low, due to a limited understanding of the molecular mechanisms underlying disease development and inadequate treatment options. $^{1,2}$ An increase in public research databases and available data has allowed for the identification of potential diagnostic biomarkers and/or therapeutic targets for GBM patients. $^{3-5}$ For example, The Cancer Genome Atlas (TCGA) offers
Correspondence: Jianjun Wang Provincial People's Hospital, Lanzhou City Republic of China

Email jianjunwang21I@I63.com

Yong Wu

Department of Gynecologic Oncology, Fudan University Shanghai Cancer Center, Shanghai, People's Republic of China Email edison-1016@163.com 
extensive cancer transcriptomic and mutation information, which contains abnormal expression information and mutation sites of key known GBM oncogenes as well as potential new oncogenic targets. ${ }^{6}$ Thus, studies on cancer transcriptome offer ways to expand our understanding of GBM development and therapeutic targets.

RNA-binding proteins (RBPs) regulate the cellular transcriptome via post-transcriptional processing of RNA transcripts, maintaining cellular homeostasis. ${ }^{7}$ To date, over 1500 RBPs have been discovered and annotated. ${ }^{8}$ Abnormal expression of RBPs has been reported in many diseases, including cancer. $^{9-11}$ For example, SERBP1 is upregulated in GBM patients and is correlated with poor survival. It controls methionine production, thereby influencing cancer metabolism and cancer epigenetics. ${ }^{12}$ Serine- and arginine-rich splicing factor 3 (SRSF3) is a key factor in the spliceosome that participates in alternative splicing. In glioma, SRSF3 is overexpressed and enhances tumorigenesis by controlling transcription factor ETS variant 1 (ETV1) and nudE neurodevelopment protein 1 (NDE1) splicing in GBM cells. ${ }^{13}$ These important findings emphasize the crucial role of RBPs in GBM development. However, their molecular functions, including the proliferative role of RBPs in GBM, remain unclear.

Aly/REF export factor ( $A L Y R E F$ ) plays key roles in RNA metabolism, and is involved in RNA nuclear export, RNA stability, and gene transcription. ${ }^{14-17}$ Specifically, ALYREF couples with RNA helicase UAP56 and the THO sub-complex to form the TREX complex that regulates the nuclear export of mRNAs. ${ }^{18}$ Recent studies have revealed that $A L Y R E F$ is dysregulated in human cancers and is correlated to poor survival. ${ }^{19}$ Saito et al identified that inhibition of $A L Y R E F$ expression resulted in cancer cell migration in human oral squamous cell carcinoma. ${ }^{20}$ To date, the detailed molecular function of $A L Y R E F$ in GBM is poorly understood.

The aim of the present study was to evaluate RBP mRNA expression in GBM, and better understand the biological role of these proteins in GBM. The up-regulation of $A L Y R E F$ is controlled by $M Y C$ at transcriptional levels. Importantly, $A L Y R E F$ drives GBM cells proliferation by enhancing the Wnt/ $\beta$-catenin signaling pathway and stabilizing $M Y C$ mRNA. Our findings suggested that an ALYREF-MYC positive feedback loop might be a potential therapeutic target for GBM patients.

\section{Materials and Methods}

\section{Tissue Sample Collection}

Cohort 1 of GBM tissues and normal brain tissues was obtained from Fudan University Shanghai Cancer Center. All tissue specimens were collected and stored at $-80^{\circ} \mathrm{C}$. Written informed consent was obtained from all patients in accordance with institutional guidelines. The study was conducted in accordance with the Declaration of Helsinki, and approved by the Ethics Committee of Fudan University Shanghai Cancer Center (approval No. 050432-4-1911D). The clinicopathological features of all patients are summarized in Table 1.

\section{Cell Culture}

HEK-293T, GBM cell line U251 and U-118 MG cells were purchased from the Shanghai Cell Bank Type Culture Collection Committee (Shanghai, China). All cells were cultured in Dulbecco's modified Eagle's medium (DMEM) supplemented with 10\% fetal bovine serum, $100 \mathrm{U} / \mathrm{mL}$ penicillin and $100 \mu \mathrm{g} / \mathrm{mL}$ streptomycin, and maintained in a cell incubator (at $37^{\circ} \mathrm{C}$ and $5 \% \mathrm{CO}_{2}$ ). Approximately $1 \times 10^{7} \mathrm{GBM}$ cells were cultured in a $10 \mathrm{~cm}$ dish for ChIP and CLIP assay. Approximately 2000 GBM cells were seeded in a 6-well plate for colony formation assay and in a 96-well plate for cell counting kit-8 assay.

Table I Correlation Between the ALYREF mRNA Expression and Clinicopathological Features

\begin{tabular}{|c|l|l|l|l|}
\hline Features & Number (n) & \multicolumn{2}{|l|}{$\begin{array}{l}\text { ALYREF Expression } \\
\text { Low (n=33) High } \\
(\mathbf{n = 3 2})\end{array}$} & P-value \\
\hline $\begin{array}{c}\text { Gender } \\
\text { Male } \\
\text { Female }\end{array}$ & 37 & 20 & 17 & 0.5426 \\
\hline $\begin{array}{c}\text { Age } \\
<55\end{array}$ & 28 & 13 & 15 & \\
$\geq 55$ & 36 & 13 & 16 & 0.3898 \\
\hline $\begin{array}{c}\text { Tumor size } \\
<5 \mathrm{~cm} \\
\geq 5 \mathrm{~cm}\end{array}$ & 34 & 20 & 16 & \\
\hline $\begin{array}{c}\text { IDHI mutation } \\
\text { Wild type }\end{array}$ & 30 & 22 & 12 & 0.0186 \\
Mutant & 35 & 11 & 20 & \\
\hline
\end{tabular}




\section{RNA Interference}

Small interfering RNAs (siRNAs) were acquired from Sangon (Shanghai, China) and transfected using Lipofectamine RNAiMAX reagent (Invitrogen). GBM cells seeded into 6-well plates were collected to validate the knockdown efficiency after $48 \mathrm{~h}$ of transfection. The targeting ALYREF siRNA sequences are listed in Supporting Table S1.

\section{Total RNA Extraction and Follow-Up}

Total RNA was extracted using the RNeasy mini kit (Qiagen) and transcribed using the PrimeScript RT Reagent Kit (TaKaRa, Shiga, Japan). qRT-PCR was performed with SYBR Premix Ex Taq II (TaKaRa) and detected using an ABI Prism 7900HT detection system (Thermo Fisher Scientific). Primer sequences of the targeted genes are listed in Supporting Table S1.

\section{Lentivirus Generation}

The GFP and MYC overexpression plasmids and the Cas9 and sgRNA plasmids targeting ALYREF were mixed with psPAX2 and pMD2.G, and co-transfected into HEK-293T cells using LipoFiter 3.0 (Hanbio, China). Lentiviruses were collected after $48 \mathrm{~h}$ and infected into GBM cells with polybrene (Sigma).

\section{Colony Formation}

Colony formation assay was performed as previously described. ${ }^{21}$ GBM cells were digested and maintained in 6 -well plates for nearly 12 days. The colonies were stained with staining buffer $(100 \%$ methanol with $0.25 \%$ crystal violet) and counted using ImageJ software.

\section{Cell Counting Kit-8 Assay}

GBM cell line U251 and U-118 MG cells were transfected with ALYREF siRNAs or infected with ALYREF sgRNA, digested and cultured in 96-well plates for 5days. Cell counting kit-8 assay was performed using Cell Counting Kit (CCK)-8 (Dojindo, Kumamoto, Japan). The OD $_{450}$ values were detected and analyzed using Biotek (USA).

\section{Xenograft in Nude Mice}

ALYREF knockdown and cas9 GBM cells were subcutaneously injected into mice (six in each group). After 25 days, the mice were sacrificed to measure tumor volume and weight. The xenograft experiment was approved and treated humanely according to the Fudan University Animal Ethics Committee (approval No. 2019-JS-058).

\section{Dual-Luciferase Assay}

The ALYREF promoter and MYC 3'-UTR sequences were inserted into the pGL3-promoter reporter plasmids (Promega, USA). GBM cells were transfected with ALYREF siRNA, GFP, MYC overexpression plasmids and reporter plasmids and were lysed with PLB buffer. Luciferase values were measured using BioTek (USA).

\section{Western Blot Analysis}

Western blot analysis was performed as previously described. ${ }^{21}$ In brief, the nitrocellulose membrane was incubated with ALYREF, Flag-tag and ACTB antibodies overnight at $4{ }^{\circ} \mathrm{C}$. The nitrocellulose membrane was washed three times and incubated with horseradish peroxidase-conjugated secondary antibodies. Detailed information on the antibodies is provided in Supporting Table S2.

\section{UV Crosslinking and Immunoprecipitation (CLIP)}

CLIP assays were carried out as previously described. ${ }^{22}$ In brief, GBM cells were irradiated at $150 \mathrm{~mJ} / \mathrm{cm}^{2}$ at $254 \mathrm{~nm}$ and lysed in CLIP lysis buffer (30 mM Tris- $\mathrm{HCl}, \mathrm{pH} 7.4$, $200 \mathrm{mM} \mathrm{NaCl}, 0.4 \% \mathrm{NP}-40,0.15 \% \mathrm{SDS}$ ) for $20 \mathrm{~min}$ on ice, digested with RNase I for $3 \mathrm{~min}$ at $37^{\circ} \mathrm{C}$, and then centrifuged at 10,000 rpm for $10 \mathrm{~min}$. The supernatant was incubated with Protein G Dynabeads (Thermo Fisher Scientific) and anti-ALYREF antibodies at $4^{\circ} \mathrm{C}$ for 6 h. Beads were collected and washed three times with CLIP lysis buffer, and then RNA was extracted using an RNeasy mini kit. Primers targeting $M Y C$ mRNA are listed in Supporting Table S1.

\section{Chromatin Immunoprecipitation (ChIP)}

ChIP assays were performed as previously described. ${ }^{21}$ In brief, GBM cells were crosslinked, lysed in ChIP lysis buffer $(40 \mathrm{mM}$ Tris- $\mathrm{HCl} \mathrm{pH} 7.5,1 \%$ NP-40, 300mM $\mathrm{NaCl}, 0.04 \%$ SDS), sonicated using a Bioruptor UCD200 (Diagenode, Liege, Belgium) and centrifuged at 13,000 rpm for 10mins. The supernatant was incubated with Protein G Dynabeads and anti-MYC antibodies at $4^{\circ}$ $\mathrm{C}$ for $6 \mathrm{~h}$. The magnetic beads were collected and washed four times. Genomic DNA was extracted using the MinElute Reaction Cleanup Kit (Qiagen). The ChIPqPCR assay was performed using the ABI Prism 7900HT detection system. Primer sequences targeting ALYREF promoters are listed in Supporting Table S1. 


\section{Statistical Analysis}

The differential expression of RBPs at the mRNA level in TCGA-GBM and GTEx datasets was conducted by moderate Student's $t$-test using R package limma. KEGG pathway analysis was performed by the DAVID platform for the upregulated ALYREF group in TCGA-GBM cohort. Gene set enrichment analysis (GSEA) of hallmark gene sets was downloaded from MSigDB database. The following R packages were used in this study: "limma", "pheatmap". The ChIP sequencing data of MYC (GEO accession: GSM822291) in HepG2 cell and the CLIP sequencing data of ALYREF (GEO Accession: GSM2631824) in HeLa cell were downloaded and analyzed from the ENCODE database (https://www.encode project.org/). All data were subjected to more than three repeats and are shown as the mean \pm SEM. Data were evaluated with the Student's $t$-test, and $\mathrm{P}<0.05$ was considered statistically significant. $* \mathrm{P}<0.05$; **P $<$ $0.01 ; * * * \mathrm{P}<0.001$.

\section{Results}

\section{ALYREF is Highly Expressed and Correlated with Proliferative Ability in GBM}

We systematically analyzed the mRNA levels of 1542 RBPs in TCGA-GBM $(n=160)$ and the Genotype-Tissue Expression (GTEx) portal normal brain tissue datasets $(\mathrm{n}=$ 222) to identify potentially dysregulated RBPs in GBM development. The expression of 448 RBPs was elevated,
A

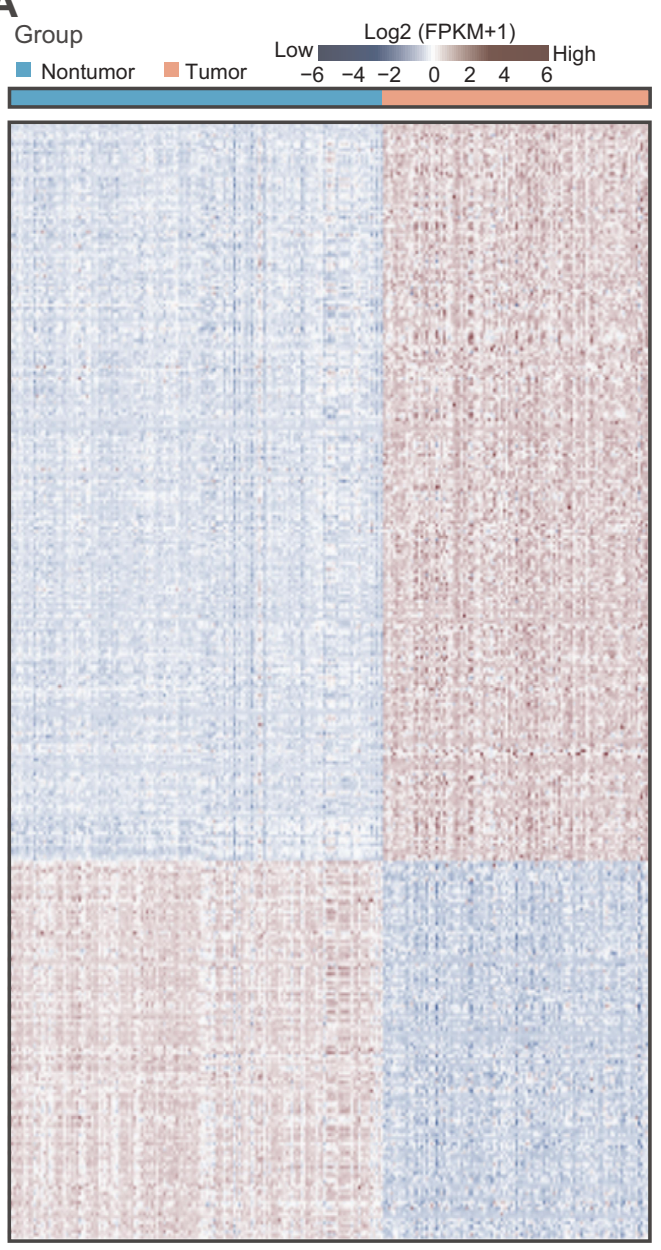

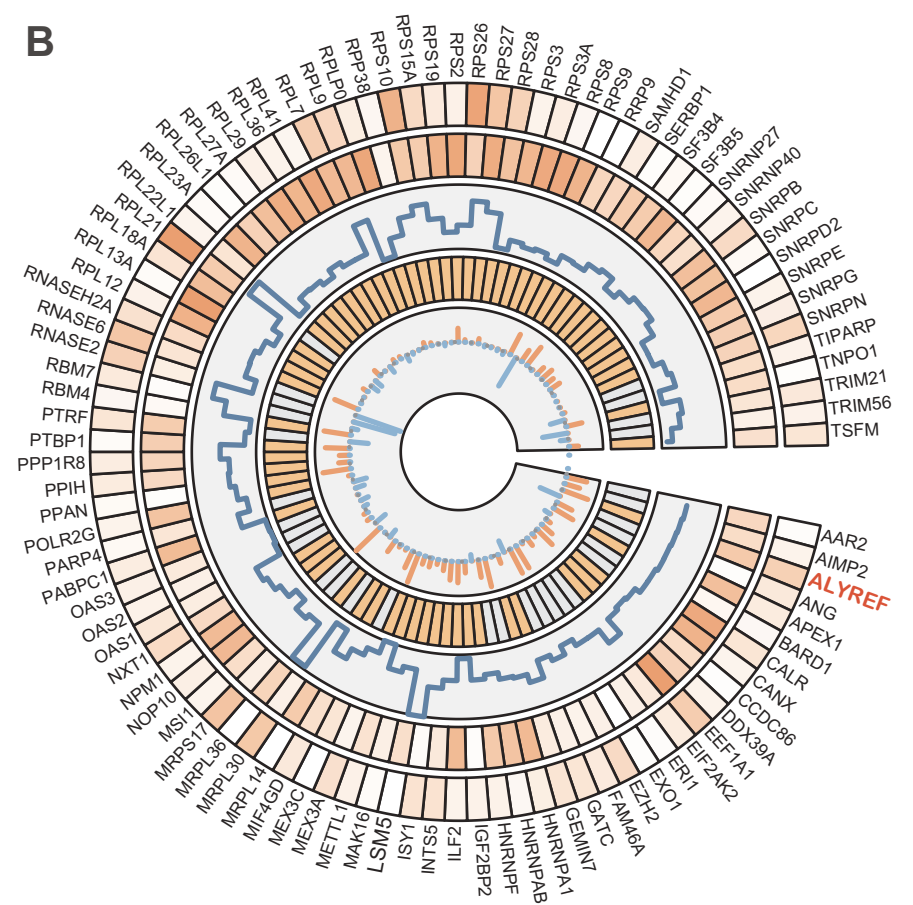
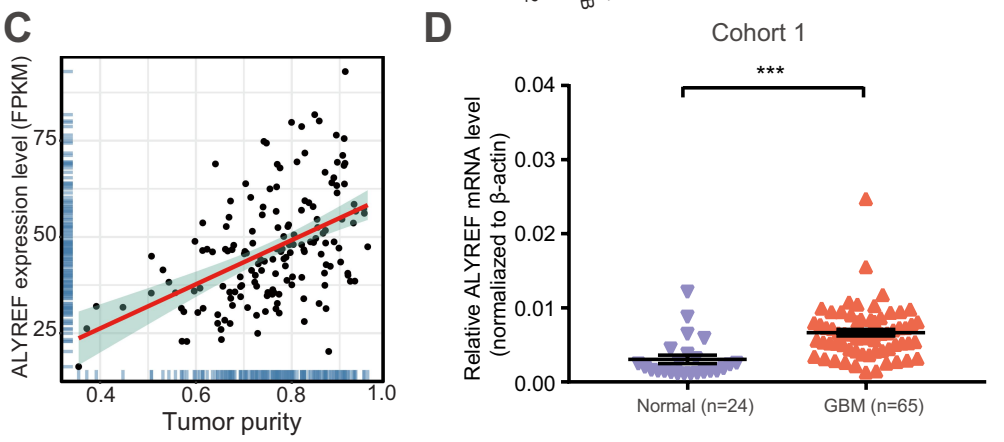

Figure I The dysregulated RBPs in GBM tissues. (A) Clustered heatmap of I542 RBPs in TCGA-GBM and GTEx cohort. (B) The circular panel represented the top upregulated 9I RBP genes. This circos plot was constructed by five tracks: (I) The expression level in normal brain tissue; (2) The expression level in GBM tissue; (3) Fold change (tumor/normal tissue); (4) Hazard ratio value; (5) The correlation between proliferative ability and mRNA levels of RBPs. (C) The correlation coefficient of ALYREF mRNA levels with tumor purity in TCGA-GBM cohort. (D) The relative mRNA levels of ALYREF in GBM and normal tissues. The results are shown as the mean \pm SEM in (D). ${ }^{* * *} \mathrm{P}<0.001$. 
and the expression of 222 RBPs was decreased (Fold change $>1.5$; Figure $1 \mathrm{~A}$ ). To reveal the potentially proliferative RBPs, we selected 91 top upregulated RBPs (Fold change $>3$ ) to analyze the proliferative correlation of upregulated RBPs in U-118 MG GBM cells (https://depmap.org, correlation coefficient $>-0.2$; Figure 1B). Among 91 RBPs, the mRNA expression levels of 10 RBPs (MSI1, MEX3A, HNRNPA1, ALYREF, PPAN, PTRF, EZH2, EXO1, RNASEH2A, PTBP1) were significantly correlated with the proliferative ability (Figure 1B). Importantly, the RBP ALYREF serves as a dominant risk factor (Hazard ratio value = 1.86). We therefore selected ALYREF for further investigation in GBM. We tested the correlation of RBPs with GBM tumor purity using the ESTIMATE deconvolution method and found that the mRNA levels of ALYREF were also strongly correlated with tumor purity in GBM tissues $(\mathrm{P}<$ $0.001, \mathrm{R}=0.48$; Figure $1 \mathrm{C}$ ). We tested the expression pattern of ALYREF in our internal GBM tissue sample cohort. The results showed that $A L Y R E F$ was significantly upregulated in GBM tissues ( $\mathrm{P}<0.001$; Figure 1D). These findings suggested that the expression of RBPs was dysregulated in GBM tissues. Among them, ALYREF serves as a potentially oncogenic regulator in GBM development.

\section{MYC Controls ALYREF mRNA Expression at the Transcriptional Levels}

To better understand the high expression levels of $A L Y R E F$ in GBM, we analyzed potential key transcriptional factors that bind to the ALYREF promoter. Importantly, we found that $M Y C$ was enriched in the core ALYREF promoter in HepG2 cells (Figure 2A). MYC is a key transcriptional factor in human cells, which encodes a protein that is coupled with MAX to control gene expression at the transcriptional level. In GBM, MYC is highly expressed and serves as an oncogene to accelerate GBM development. ${ }^{23}$ We hypothesized that $M Y C$ may also regulate ALYREF at the transcriptional level in GBM cells. Therefore, we performed ChIP-qPCR, and the results showed similar binding ability in GBM cells (Figure 2B). To further validate this regulatory process, we performed dual-luciferase and found that overexpression of $M Y C$ enhanced ALYREF promoter activity in GBM cells (Figure 2C). Overexpression of $M Y C$ also upregulated ALYREF expression in $\mathrm{U} 251$ and $\mathrm{U}-118 \mathrm{MG}$ cells (Figure 2D). These findings showed that high expression of $A L Y R E F$ in GBM is regulated by $M Y C$ at the RNA level.

A

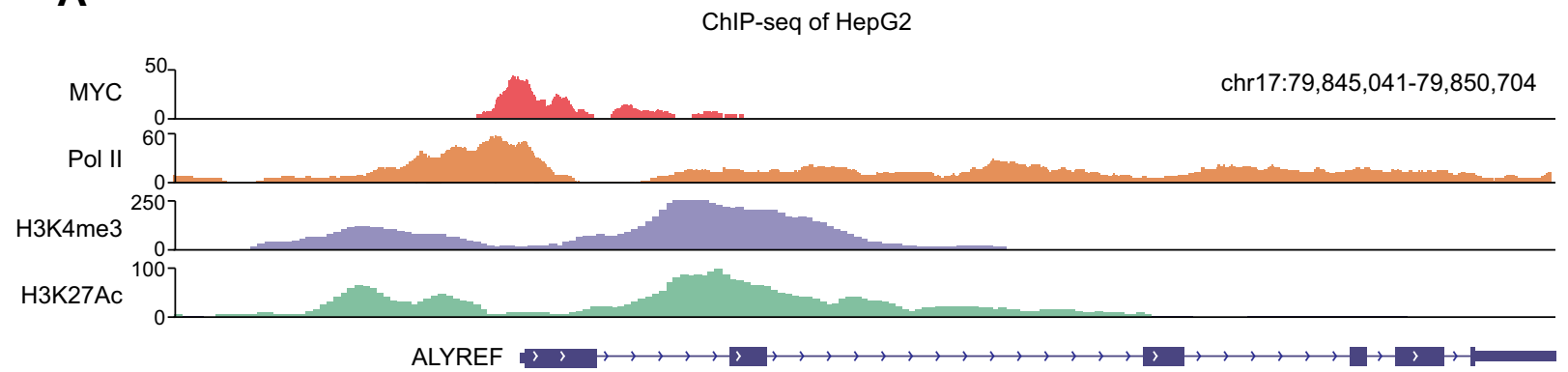

B

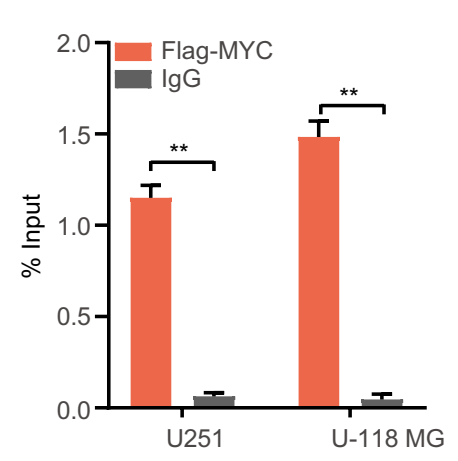

C

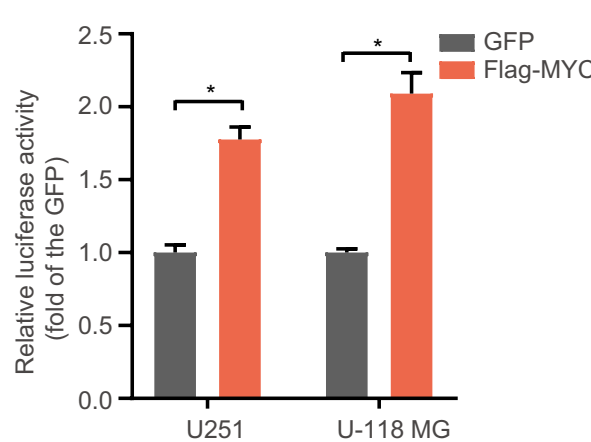

D

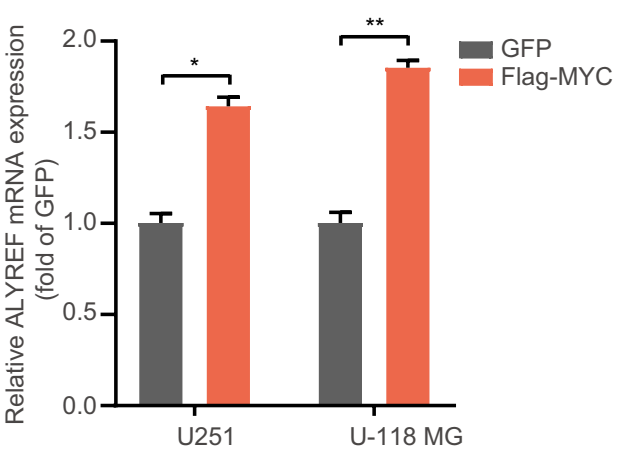

Figure 2 ALYREF is regulated by MYC at RNA levels. (A) The ChIP sequencing data showed the enrichment of MYC, Polll, H3K4me3 and H3K27Ac signaling at ALYREF promoter in HepG2 cells. (B) ChIP-qPCR validation for MYC at ALYREF promoter in GBM cells. (C) U25I and U-II8 MG cells were transfected with GFP or MYC overexpression plasmids, and the relative luciferase activity of ALYREF promoter was measured. (D) The relative ALYREF mRNA levels transfected with GFP or MYC overexpression plasmids in U25I and U-II8 MG cells. The results are shown as the mean \pm SEM, $n=3$ in $(\mathbf{B}-\mathbf{D})$. *P $<0.05$; **P $<0.01$. 


\section{ALYREF Promotes GBM Cells}

\section{Proliferation}

To further explore the proliferative effects of $A L Y R E F$ in GBM cells, we first knocked-down ALYREF expression with specific siRNAs. The knockdown efficiency in GBM cells was determined by Western blot analysis (Figure $\underline{\mathrm{S} 1 \mathrm{~A}}$ ). Proliferation assays demonstrated that inhibition of ALYREF dramatically downregulated GBM cell proliferation and colony formation in vitro (Figure $3 \mathrm{~A}$ and $\mathrm{B}$ ). Next, we used the CRISPR tool to knock down ALYREF (Figure S1B), which showed identical molecular function to the siRNA interference experiment (Figure 3C and D). Finally, we infected Cas 9 and sgRNA lentiviruses targeting ALYREF in U251 cells and performed a xenograft experiment to evaluate the proliferative property in vivo. Importantly, inhibition of ALYREF expression dramatically abolished tumorigenicity (Figure $3 \mathrm{E}-\mathrm{G}$ ). These results suggested that ALYREF accelerated GBM cell proliferation both in vitro and in vivo.

\section{ALYREF Regulates the $\mathrm{Wnt} / \beta$-Catenin Signaling Pathway}

Our previous studies showed that ALYREF served as an oncogene in GBM development. To understand its oncogenic mechanism in GBM, we first separated GBM samples from TCGA datasets into high and low ALYREF-expression groups. Second, we analyzed the cancer hallmarks enriched in the ALYREF high-expression group using single sample gene set enrichment method (ssGSEA). The results showed that some key cancer hallmarks, such as the Wnt/ $\beta$-catenin signaling pathway, MYC targets, and NOTCH signaling pathway were activated in the ALYREF high-expression group (Figure 4A). GSEA analysis showed that the Wnt/ $\beta$ catenin signaling pathway, MYC targets, G2M checkpoints,
A
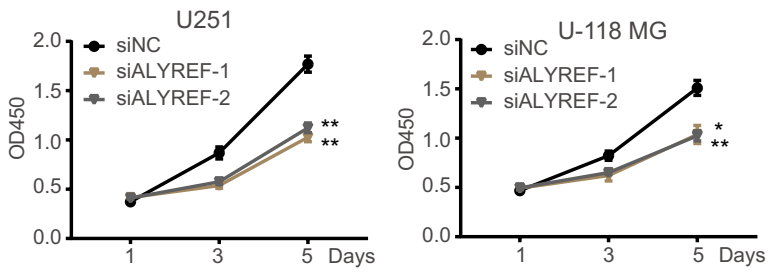

C
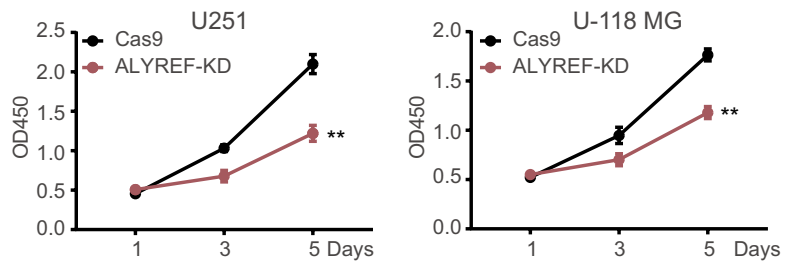

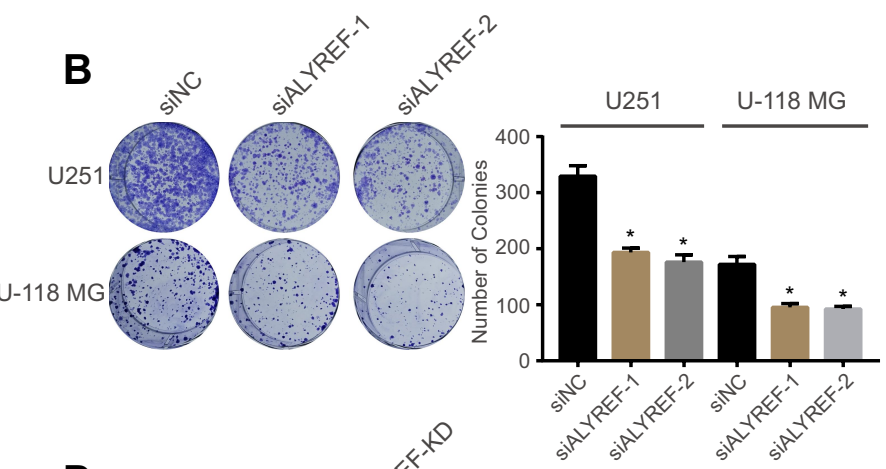

E

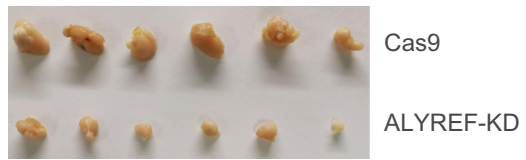

$\mathbf{F}$

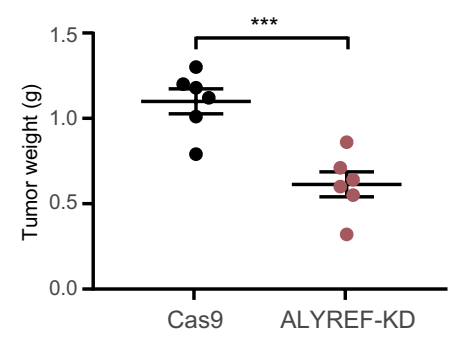

D
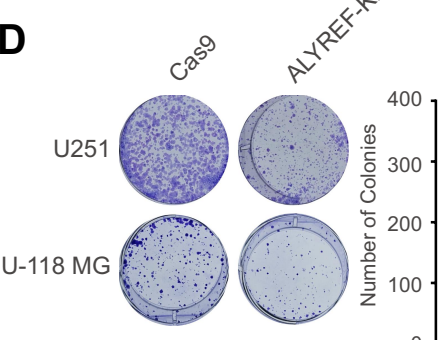

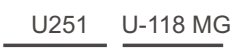

\section{G}

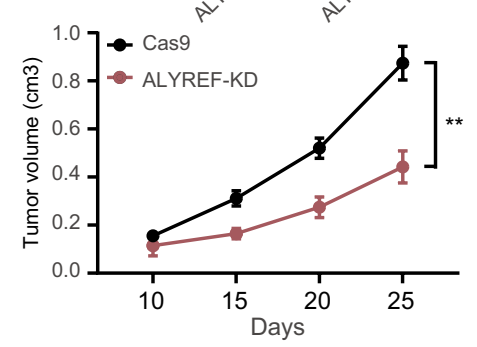

Figure 3 ALYREF promotes GBM cells growth and tumor formation. (A, B) U25I and U-II8 MG cells transfected with ALYREF siRNAs or control siRNA, and the cell counting kit-8 assay (A) and colony formation assay (B) were determined. (C, D) U25I and U-I I8 MG cells infected with cas9 and ALYREF sgRNA or control sgRNA to perform cell counting kit-8 assay (C) and colony formation assay (D). (E) Tumor formation of Cas9 or ALYREF knockdown U25I cells were performed. (F, G) The tumor weight $(\mathbf{F})$ and tumor volume $(\mathbf{G})$ of $U 25 I$ cells infected with Cas9 and ALYREF sgRNA or control sgRNA. Data $(n=3)$ was represented as the mean \pm SEM in $(\mathbf{A}-\mathbf{D} ; \mathbf{F}, \mathbf{G})$. $* \mathrm{P}<0.05$; **P $<0.01$; *** $\mathrm{P}<0.00 \mathrm{I}$. 

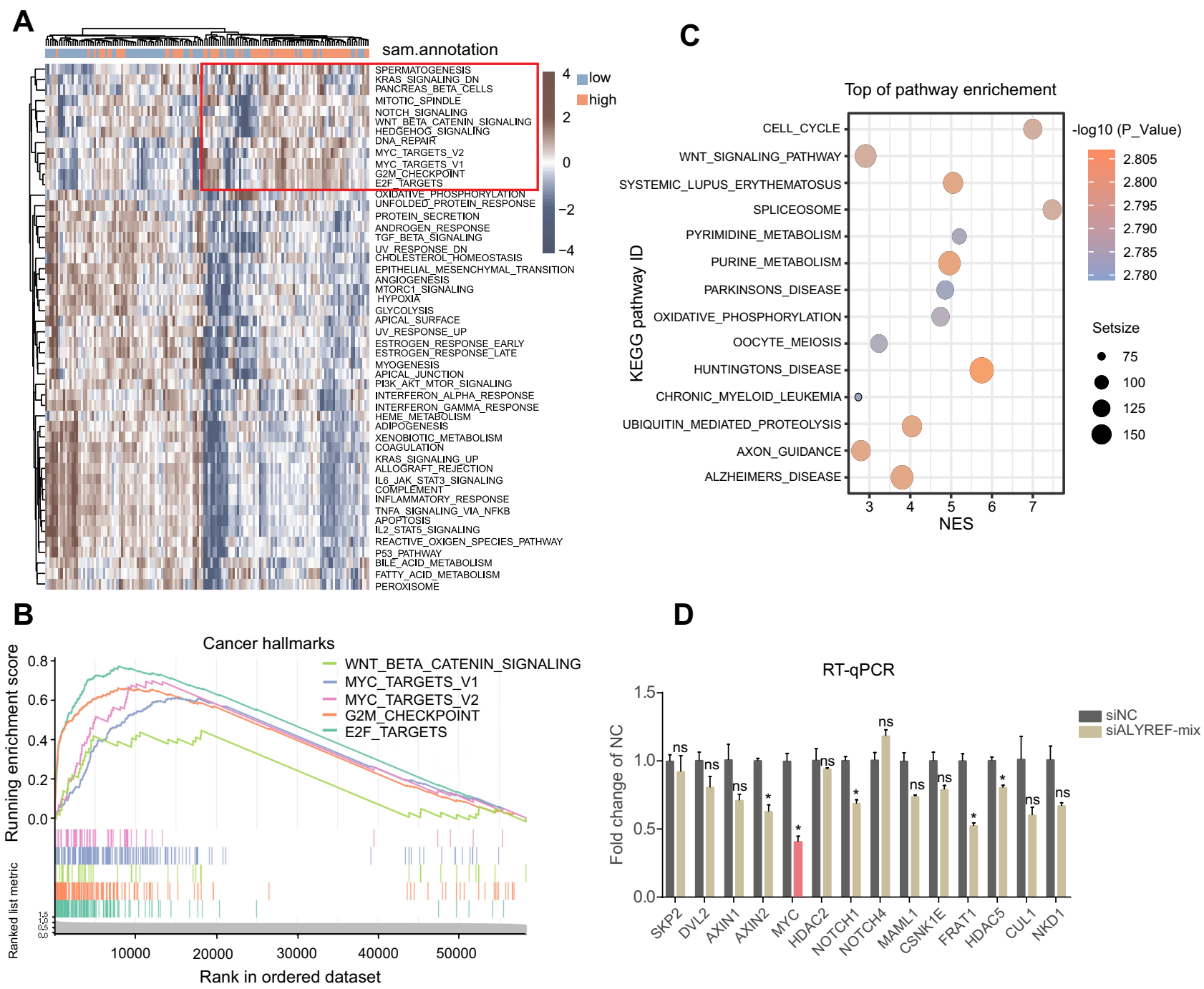

Figure 4 ALYREF activates Wnt/ $\beta$-catenin signaling pathway and control MYC mRNA levels. (A) The heatmap represents the cancer hallmarks enriched in high or lowALYREF expressional group of TCGA-GBM cohort. (B) GSEA analysis for cancer hallmarks in ALYREF high expressed group of TCGA-GBM cohort. (C) KEGG pathway analysis for upregulated ALYREF group in TCGA-GBM cohort. (D) The relative target genes mRNA levels of Wnt/ $\beta$-catenin signaling pathway transfected with control or ALYREF siRNAs in GBM cell. Data $(n=3)$ was represented as the mean \pm SEM in $(\mathbf{D}) . * P<0.05$.

and E2F targets were significantly enriched (enrichment score $>0, \mathrm{P}<0.05$; Figure $4 \mathrm{~B}$ ). We also found that the Wnt/ $\beta$-catenin and cell cycle signaling pathways were enriched in the high ALYREF sub-group through KEGG analysis (Figure 4C). To validate the enrichment of signaling pathways in the $A L Y R E F$ high-expression group, we selected the top 14 genes in the $\mathrm{Wnt} / \beta$-catenin pathway which their expression was correlated with ALYREF mRNA levels ( $\mathrm{R}>$ $0.3, \mathrm{P}<0.05)$ and performed RT-qPCR analysis. The results showed that genes like AXIN2 and FRAT1 were decreased upon inhibition of $A L Y R E F$ expression. Importantly, the mRNA level of $M Y C$ was dramatically downregulated in GBM cells when $A L Y R E F$ was knocked-down (Figure 4D).
These studies demonstrated that ALYREF drives GBM development by activating the $\mathrm{Wnt} / \beta$-catenin pathway.

\section{ALYREF Enhances MYC mRNA Stability in GBM Cells}

To further identify the detailed mechanism by which $A L Y R E F$ regulates $M Y C$ mRNA levels in GBM cells, we searched public CLIP sequencing data and found that $A L Y R E F$ is an $\mathrm{RBP}$ that binds specifically to $M Y C \mathrm{mRNA}$, notably in the 3'UTR region of HeLa cells (Figure 5A). Previous studies have reported that the $3^{\prime}$-UTR region of human genes is the key sequence regulating mRNA stability in human cancers. ${ }^{24,25}$ Therefore, we speculated that $A L Y R E F$ binds to the 3 '-UTR of 
MYC mRNA to control its stability, and drive GBM cell proliferation. To test this, we first performed CLIP-qPCR analysis and found that $A L Y R E F$ bound to MYC mRNA in GBM cells (Figure 5B). Next, we constructed 3'-UTR reporter plasmids of $M Y C$ and found that knockdown of $A L Y R E F$ decreased luciferase activity of the $M Y C$ 3'-UTR sequence (Figure 5C). Knockdown of ALYREF also reduced $M Y C$ mRNA stability in GBM cells (Figure 5D). Importantly, overexpression of $M Y C$ could rescue the proliferative ability of ALYREF-deficient GBM cells (Figure 5E). Finally, we analyzed TCGA expression data and found that the mRNA expression of $A L Y R E F$ was positively correlated with $M Y C$ mRNA in GBM samples (Figure 5F). These findings indicate that $A L Y R E F$ promotes carcinogenesis by controlling MYC mRNA stability in GBM cells.

\section{Discussion}

RBPs have recently been reported to be dysregulated in many cancer tissues. The oncogenic RBPs not only participate in cancer initiation but also control cancer progression. $^{26,27}$ Moreover, targeting these RBPs may have potential clinical application in cancer treatment. ${ }^{28}$

\section{A}

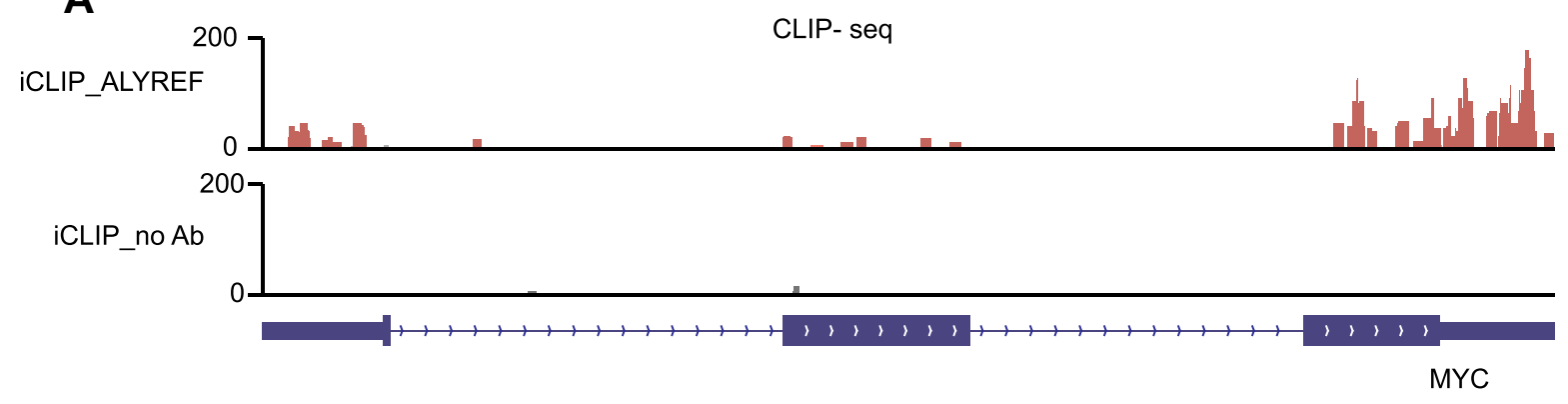

B

iCLIP-qPCR

C

Luciferase activity

D

RNA stabliity

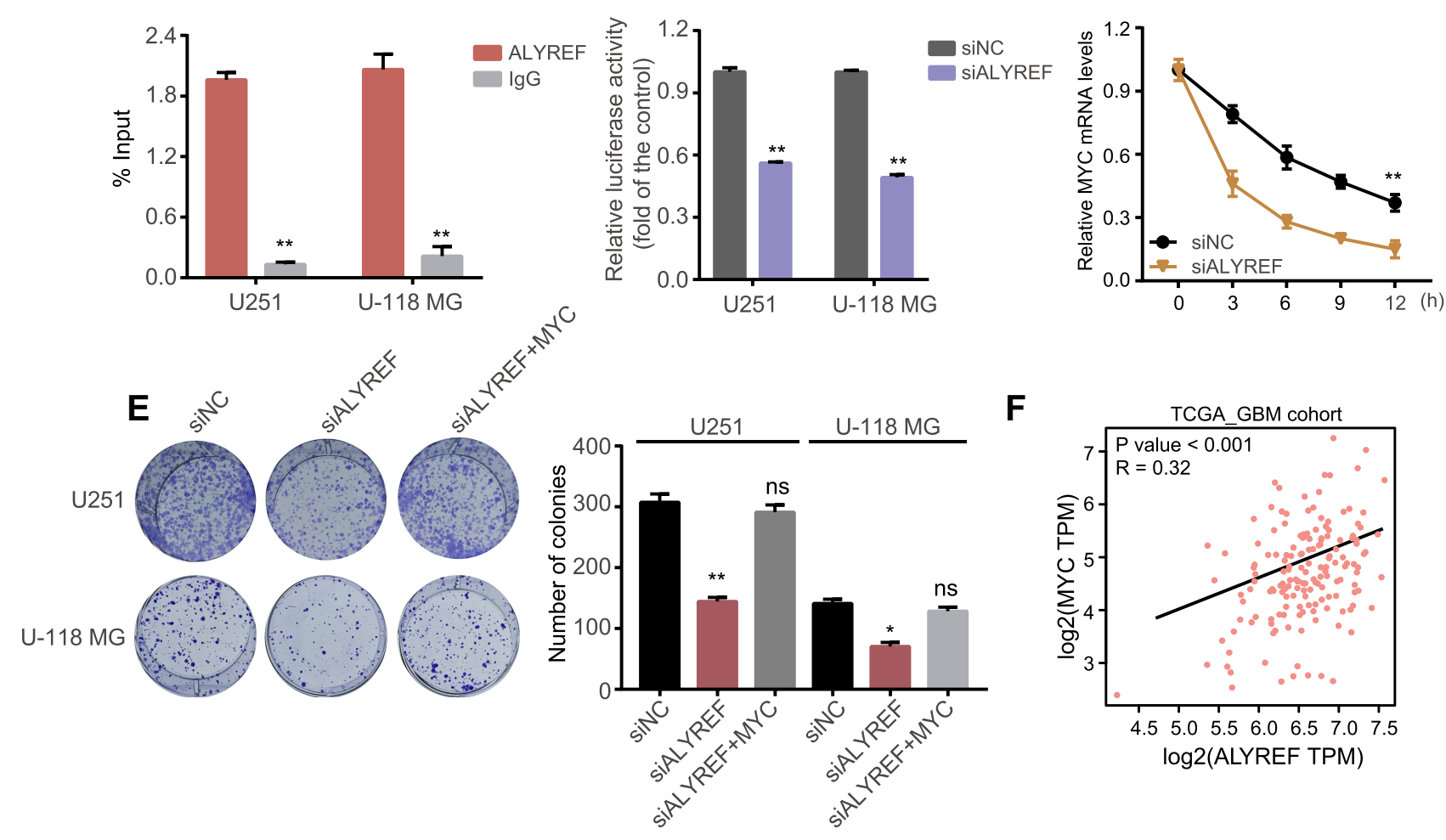

Figure 5 ALYREF controls MYC mRNA stability in GBM cells. (A) The CLIP sequencing data showed the enrichment of ALYREF at 3'-UTR of MYC in HeLa cell. (B) iCLIPqPCR validation for ALYREF binding to MYC mRNA in GBM cells. (C) The relative luciferase activity of 3'-UTR of MYC transfected with control or ALYREF siRNAs in U25I and U-I I8 MG cells. (D) The relative MYC mRNA levels in U25I and U-II8 MG cells transfected with control or ALYREF siRNAs and treated with actinomycin D. (E) The colony assay transfected with ALYREF, ALYREF plus MYC overexpression plasmids or negative siRNA in U25I and U-II8 MG cells. (F) The expressional correlation of ALYREF with MYC in TCGA-GBM cohort. The results $(n=3)$ were represented as the mean \pm SEM in $(B-E)$. $* P<0.05 ; * * P<0.01$. 
However, the molecular mechanisms governing GBM, including the proliferative properties of this cancer, remain unclear. In this work, we analyzed public TCGA-GBM RNA sequencing data and identified a group of RBPs that were dysregulated and that potentially promoted GBM cell proliferation. Of note, we found that ALYREF is overexpressed in GBM patients and triggers GBM cell proliferation. These findings suggest that $A L Y R E F$ plays an oncogenic role in GBM development. We also assessed the expression of ALYREF in other cancers and found that it was also highly upregulated in brain lower grade glioma, breast cancer, colon cancer and liver hepatocellular carcinoma. These results suggest that ALYREF might also participate in the progression of other tumors.

To further examine the mechanisms underlying the upregulation and proliferative function of $A L Y R E F$ in GBM, we analyzed related transcriptional factors and found that MYC is enriched in the ALYREF promoter and regulates ALYREF mRNA expression. Studies have reported that $M Y C$ serves as a key transcriptional factor that enhances glioma cell proliferation and migration. ${ }^{29,30}$ We analyzed MYC mRNA levels and found that $M Y C$ is also highly expressed in GBM and brain lower grade glioma (LGG) tissues and its expression is positively correlated with ALYREF mRNA expression (data partially not shown). It has recently been reported that RPBs can regulate $M Y C$ mRNA stability. For example, Du et al found that long non-coding RNA (lncRNA) linc02042 enhanced tumorigenesis in esophageal squamous cell carcinoma by recruiting YBX1 to stabilize MYC mRNA. ${ }^{31}$ Our study revealed that $A L Y R E F$ protein could bind to and stabilize MYC mRNA, which forms a positive loop to accelerate GBM development. Interestingly, RNA modifications and their RNA-modifying proteins (RMPs) have also been identified as key players in strengthening the aggressiveness of GBM. ${ }^{32}$ Moreover, Yang et al recently reported that 5-methylcytosine $\left(\mathrm{m}^{5} \mathrm{C}\right)$ of RNA is specifically recognized by $A L Y R E F F^{33}$ How ${ }^{5} \mathrm{C}$ modification of oncogenic RNAs regulated by $A L Y R E F$ in GBM needs to be further investigated.

The findings of the present study further revealed a potential group of RBPs that participate in GBM proliferation, including $M E X 3 A, S N R P B$, and INTS5. It has been reported that $M E X 3 A$ and $S N R P B$ act as oncogenes to promote GBM tumorigenesis. ${ }^{34,35}$ In pancreatic ductal adenocarcinoma (PDAC), increased expression of MEX3A is correlated with higher disease stage, and depletion of MEX3A decreased resistance to gemcitabine for PDAC patients. ${ }^{36}$ INTS5 is a factor in the integrator complex, where it binds to the RNA polymerase II to control RNA transcription and processing. Bioinformatics analysis has found that could be abnormally expressed in specific cancers. ${ }^{37}$ In GBM, further studies investigating the detailed molecular functions and clinical significance of INTS5 should be undertaken.

\section{Conclusion}

In conclusion, our study demonstrates that ALYREF is highly expressed in some GBM patients, and is regulated by $M Y C$ at the transcriptional level. ALYREF enhances the high proliferation activity of GBM cells through the Wnt/ $\beta$-catenin signaling pathway and stabilizing $M Y C$ mRNA. The ALYREF-MYC positive feedback loop may represent a potential therapeutic target for GBM patients.

\section{Abbreviations}

RBPs, RNA-binding proteins; GBM, glioblastoma; ALYREF, Aly/REF export factor; GSEA, gene set enrichment analysis; ChIP, Chromatin immunoprecipitation; CLIP, UV crosslinking and immunoprecipitation; MYC, MYC proto-oncogene, bHLH transcription factor; TCGA, The Cancer Genome Atlas; SERBP1, SERPINE1 mRNA binding protein 1; SRSF3, serine- and arginine-rich splicing factor 3; ETV1, ETS variant 1; NDE1, nudE neurodevelopment protein 1; RMPs, RNA-modifying proteins.

\section{Data Sharing Statement}

The datasets in this study are available from the corresponding author upon reasonable request.

\section{Acknowledgments}

We are grateful for Dr Didier Trono's gifts of the psPAX2 and pMD2.G lentivirus plasmids.

\section{Author Contributions}

Jianjun Wang and Yong $\mathrm{Wu}$ designed the study; Jianjun Wang, Binbin Xu, Jiao Dong, Haiyan Zhao and Dongxia Zhao acquired the data; Jianjun Wang, Yong $\mathrm{Wu}$ and Yuchen Li analyzed the data; Jianjun Wang and Yuchen Li wrote the paper. All authors contributed to data analysis, drafting or revising the article, have agreed on the journal to which the article will be submitted, gave final approval of the version to be published, and agree to be accountable for all aspects of the work. 


\section{Disclosure}

The authors declare that they are no conflicts of interest for this work.

\section{References}

1. Liou TG, Adler FR, Cahill BC, Cox DR. Correction: lung transplantation and survival in children with cystic fibrosis. $N$ Engl J Med. 2008;359(5):536. doi:10.1056/NEJMc086289

2. Stupp R, Hegi ME, Mason WP, et al.; R. European Organisation for, T. Treatment of Cancer Brain, G. Radiation Oncology, G. National Cancer Institute of Canada Clinical Trials. Effects of radiotherapy with concomitant and adjuvant temozolomide versus radiotherapy alone on survival in glioblastoma in a randomised phase III study: 5-year analysis of the EORTC-NCIC trial. Lancet Oncol. 2009;10:459-466. doi:10.1016/S1470-2045(09)70025-7

3. Weinstein JN, Collisson EA, Mills GB, et al.; N. Cancer Genome Atlas Research. The cancer genome atlas pan-cancer analysis project. Nat Genet. 2013;45(10):1113-1120. doi:10.1038/ng.2764

4. Becker PB; E.P. Consortium. A user's guide to the encyclopedia of DNA elements (ENCODE). PLoS Biol. 2011;9(4):e1001046. doi:10.1371/journal.pbio.1001046

5. Bernstein BE, Stamatoyannopoulos JA, Costello JF, et al. The NIH roadmap epigenomics mapping consortium. Nat Biotechnol. 2010;28:1045-1048. doi:10.1038/nbt1010-1045

6. Pangeni RP, Zhang Z, Alvarez AA, et al. Genome-wide methylomic and transcriptomic analyses identify subtype-specific epigenetic signatures commonly dysregulated in glioma stem cells and glioblastoma. Epigenetics. 2018;13:432-448. doi:10.1080/ 15592294.2018.1469892

7. Luo E-C, Nathanson JL, Tan FE, et al. Large-scale tethered function assays identify factors that regulate mRNA stability and translation. Nat Struct Mol Biol. 2020;27(10):989-1000. doi:10.1038/s41594020-0477-6

8. Gerstberger S, Hafner M, Tuschl T. A census of human RNA-binding proteins. Nat Rev Genet. 2014;15(12):829-845. doi:10.1038/nrg3813

9. Montalbano M, McAllen S, Puangmalai N, et al. RNA-binding proteins musashi and tau soluble aggregates initiate nuclear dysfunction. Nat Commun. 2020;11(1):4305. doi:10.1038/s41467-020-18022-6

10. Wang ZL, Li B, Luo YX, et al. Comprehensive genomic characterization of RNA-binding proteins across human cancers. Cell Rep. 2018;22:286-298. doi:10.1016/j.celrep.2017.12.035

11. Takayama K-I, Suzuki T, Fujimura T, et al. Dysregulation of spliceosome gene expression in advanced prostate cancer by RNA-binding protein PSF. Proc Natl Acad Sci U S A. 2017;114(39):10461-10466. doi:10.1073/pnas.1706076114

12. Kosti A, de Araujo PR, Li WQ, et al. The RNA-binding protein SERBP1 functions as a novel oncogenic factor in glioblastoma by bridging cancer metabolism and epigenetic regulation. Genome Biol. 2020;21:195. doi:10.1186/s13059-020-02115-y

13. Song X, Wan X, Huang T, et al. SRSF3-regulated RNA alternative splicing promotes glioblastoma tumorigenicity by affecting multiple cellular processes. Cancer Res. 2019;79:5288-5301. doi:10.1158/ 0008-5472.CAN-19-1504

14. Fan J, Wang K, Du X, et al. ALYREF links 3 '-end processing to nuclear export of non-polyadenylated mRNAs. EMBO J. 2019;38. doi: $10.15252 / \mathrm{embj} .201899910$

15. Shi M, Zhang H, Wu X, et al. ALYREF mainly binds to the $5^{\prime}$ and the 3' regions of the mRNA in vivo. Nucleic Acids Res. 2017;45:9640-9653. doi:10.1093/nar/gkx597

16. Fan J, Kuai B, Wu G, et al. Exosome cofactor hMTR4 competes with export adaptor ALYREF to ensure balanced nuclear RNA pools for degradation and export. EMBO J. 2017;36:2870-2886. doi:10.15252/ embj.201696139
17. Stubbs SH, Conrad NK. Depletion of REF/Aly alters gene expression and reduces RNA polymerase II occupancy. Nucleic Acids Res. 2015;43(1):504-519. doi:10.1093/nar/gku1278

18. Chi B, Wang Q, Wu G, et al. Aly and THO are required for assembly of the human TREX complex and association of TREX components with the spliced mRNA. Nucleic Acids Res. 2013;41(2):1294-1306. doi:10.1093/nar/gks1188

19. Dominguez-Sanchez MS, Saez C, Japon MA, Aguilera A, Luna R. Differential expression of THOC1 and ALY mRNP biogenesis/export factors in human cancers. BMC Cancer. 2011;11(1):77. doi:10.1186/ 1471-2407-11-77

20. Saito Y, Kasamatsu A, Yamamoto A, et al. ALY as a potential contributor to metastasis in human oral squamous cell carcinoma. $J$ Cancer Res Clin Oncol. 2013;139(4):585-594. doi:10.1007/ s00432-012-1361-5

21. Shang Y, Li Y, Zhang Y, Wang J. ZNF436 promotes tumor cell proliferation through transcriptional activation of BCL10 in glioma. Biochem Biophys Res Commun. 2019;515(4):572-578. doi:10.1016/j. bbrc.2019.06.004

22. Gajduskova P, Ruiz de Los Mozos I, Rajecky M, Hluchy M, Ule J, Blazek D. CDK11 is required for transcription of replication-dependent histone genes. Nat Struct Mol Biol. 2020;27 (5):500-510. doi:10.1038/s41594-020-0406-8

23. Wang G, Wang J, Zhao H, Wang J, Tony SS. To, the role of Myc and let-7a in glioblastoma, glucose metabolism and response to therapy. Arch Biochem Biophys. 2015;580:84-92. doi:10.1016/j. abb.2015.07.005

24. Li F, Zhao H, Su M, et al. HnRNP-F regulates EMT in bladder cancer by mediating the stabilization of snaill mRNA by binding to its $3^{\prime}$ UTR. EBioMedicine. 2019;45:208-219. doi:10.1016/j. ebiom.2019.06.017

25. Kataoka K, Shiraishi Y, Takeda Y, et al. Aberrant PD-L1 expression through $3^{\prime}$-UTR disruption in multiple cancers. Nature. 2016;534:402-406. doi:10.1038/nature18294

26. Li X, Qian X, Peng LX, et al. A splicing switch from ketohexokinase-C to ketohexokinase-A drives hepatocellular carcinoma formation. Nat Cell Biol. 2016;18:561-571. doi:10.1038/ ncb3338

27. Lovnicki J, Gan Y, Feng T, et al. LIN28B promotes the development of neuroendocrine prostate cancer. J Clin Invest. 2020;130 (10):5338-5348. doi:10.1172/JCI135373

28. Mohibi S, Chen X, Zhang J. Cancer the'RBP'eutics-RNA-binding proteins as therapeutic targets for cancer. Pharmacol Ther. 2019;203:107390. doi:10.1016/j.pharmthera.2019.07.001

29. Ning JF, Stanciu M, Humphrey MR, et al. Myc targeted CDK18 promotes ATR and homologous recombination to mediate PARP inhibitor resistance in glioblastoma. Nat Commun. 2019;10:2910. doi:10.1038/s41467-019-10993-5

30. Zhao K, Wang Q, Wang Y, et al. EGFR/c-myc axis regulates TGFbeta/hippo/notch pathway via epigenetic silencing miR-524 in gliomas. Cancer Lett. 2017;406:12-21. doi:10.1016/j. canlet.2017.07.022

31. Du J, Zhang G, Qiu H, Yu H, Yuan W. A novel positive feedback loop of linc 02042 and c-Myc mediated by YBX1 promotes tumorigenesis and metastasis in esophageal squamous cell carcinoma. Cancer Cell Int. 2020;20(1):75. doi:10.1186/s12935-020-1154-x

32. Dong Z, Cui H. The emerging roles of RNA modifications in glioblastoma. Cancers. 2020;12(3):736. doi:10.3390/ cancers 12030736

33. Yang X, Yang Y, Sun B-F, et al. 5-methylcytosine promotes mRNA export - NSUN2 as the methyltransferase and ALYREF as an m5C reader. Cell Res. 2017;27(5):606-625. doi:10.1038/cr.2017.55

34. Bufalieri F, Caimano M, Lospinoso Severini L, et al. The RNA-binding ubiquitin ligase MEX3A affects glioblastoma tumorigenesis by inducing ubiquitylation and degradation of RIG-I. Cancers. 2020;12(2):321. doi:10.3390/cancers12020321 
35. Correa BR, de Araujo PR, Qiao M, et al. Functional genomics analyses of RNA-binding proteins reveal the splicing regulator SNRPB as an oncogenic candidate in glioblastoma. Genome Biol. 2016;17:125. doi:10.1186/s13059-016-0990-4

36. Panzeri V, Manni I, Capone A, et al. The RNA-binding protein $\mathrm{MEX} 3 \mathrm{~A}$ is a prognostic factor and regulator of resistance to gemcitabine in pancreatic ductal adenocarcinoma. Mol Oncol. 2020. doi:10.1002/1878-0261.12847
37. Federico A, Rienzo M, Abbondanza C, Costa V, Ciccodicola A, Casamassimi A. Pan-cancer mutational and transcriptional analysis of the integrator complex. Int J Mol Sci. 2017;18. doi:10.3390/ ijms 18050936

\section{Publish your work in this journal}

OncoTargets and Therapy is an international, peer-reviewed, open access journal focusing on the pathological basis of all cancers, potential targets for therapy and treatment protocols employed to improve the management of cancer patients. The journal also focuses on the impact of management programs and new therapeutic

Submit your manuscript here: https://www.dovepress.com/oncotargets-and-therapy-journal agents and protocols on patient perspectives such as quality of life, adherence and satisfaction. The manuscript management system is completely online and includes a very quick and fair peer-review system, which is all easy to use. Visit http://www.dovepress.com/ testimonials.php to read real quotes from published authors. 